\title{
NADPH oxidase 1 inhibition attenuates platelet activation and thrombosis in vivo without affecting haemostasis
}

\author{
Dina Vara ${ }^{1}$, Anuradha Tarafdar ${ }^{2}$, Meral Celikag ${ }^{1}$, Daniela Patina ${ }^{1}$, Christina Gulacsy ${ }^{3}$, \\ Eleanor Hounslea ${ }^{3}$, Zach Warren ${ }^{1}$, Bárbara Ferreira ${ }^{1}$, Maarten Koeners ${ }^{1}$, Lorenzo \\ Caggiano $^{3}$, and Giordano Pula ${ }^{4}$ \\ ${ }^{1}$ University of Exeter \\ ${ }^{2}$ The University of Manchester \\ ${ }^{3}$ University of Bath \\ ${ }^{4}$ University Medical Center Hamburg-Eppendorf
}

April 28, 2020

\begin{abstract}
Background and Purpose - Growing evidence supports a central role of NADPH oxidases (NOXs) in the regulation of platelets. Experimental Approach - Here, we characterise the NOX inhibitor 2-acetylphenothiazine (2APT) and nine of its chemical derivatives for their selectivity, their effect on platelet activation in response to different stimuli ex vivo, and their modulation of carotid thrombosis and hemostasis in vivo. Key Results - Using Nox1-/- mice, we proved that NOX1 is critical for collagendependent platelet aggregation and carotid thrombosis, while it does not affect thrombin-dependent aggregation or haemostasis. 2APT selectively inhibits NOX1 over NOX2 (IC50 $141 \mathrm{nM}$ and $>10 \mu \mathrm{M}$, respectively). In agreement with a central role of NOX1 in collagen signalling, 2APT and its most potent derivative 1-(10H-phenothiazin-2-yl)vinyl tert-butyl carbonate (or 2APT-D6) inhibit collagen-dependent platelet aggregation with negligible effects on thrombin responses. 2APT-D6 displays higher potency compared to $2 \mathrm{APT}$ (52 vs $141 \mathrm{nM}$, respectively). Platelet adhesion to collagen in static and flow conditions, superoxide anion generation and surface maker expression in response to collagen were also inhibited by 2APT and 2APT-D6. Administration of 2APT or 2APT-D6 $(200 \mathrm{mg} / \mathrm{kg})$ in diet for 48 hours led to inhibition of platelet aggregation, oxygen radical output, and thrombus formation, and carotid occlusion in vivo, while tail hemostasis was not affected. Conclusion and Implications - In summary, this study suggests that NOX1 inhibition by 2APT or 2APT-D6 is a viable strategy to control collagen-induced platelet activation and reduce thrombosis in vivo without acute deleterious effects on hemostasis or other aspects of mouse health.
\end{abstract}

\section{Introduction}

Important vascular diseases such as stroke, coronary artery disease (CAD) and peripheral artery disease (PAD) are caused by platelet hyperactivity. Dysregulated generation of reactive oxygen species (ROS) causes platelet hyperactivity (El Haouari, 2019; Ghasemzadeh, Hosseini, Roudsari \& Zadkhak, 2018; Krotz et al., 2002; Monteiro et al., 2012) and NADPH oxidases (NOXs) are important sources of ROS in platelets (Begonja et al., 2005; Gray et al., 2013; Magwenzi et al., 2015). Both NOX1 and NOX2 are the main NADPH oxidases expressed in human platelets (Vara, Campanella \& Pula, 2013). Although the differential roles of these two enzymes in platelet regulation remain unclear (Abubaker, Vara, Eggleston, Canobbio \& Pula, 2019; Delaney et al., 2016; Sonkar et al., 2019; Walsh, Berndt, Carrim, Cowman, Kenny \& Metharom, 2014), a convincing amount of evidence suggests that the enzymatic activity of NOXs is necessary for platelet responses (Carnevale et al., 2014; Chlopicki, Olszanecki, Janiszewski, Laurindo, Panz \& Miedzobrodzki, 2004). Novel pharmacological tools for the inhibition of NOXs have been developed in recent years (Anvari, Wikstrom, 
Walum \& Welsh, 2015; Gianni et al., 2010; Hirano et al., 2015; Jiang et al., 2012; Kwon et al., 2017; Vara, Campanella \& Pula, 2013; Wingler, Altenhoefer, Kleikers, Radermacher, Kleinschnitz \& Schmidt, 2012), which could therefore be investigated for conditions characterized by platelet hyperactivity, such as diabetes, hypertension, and atherosclerosis (Dayal, Wilson, Motto, Miller, Chauhan \& Lentz, 2013; El Haouari, 2019; Gray et al., 2013; Magwenzi et al., 2015). Interestingly, the efficacy of NOX inhibition in patients displaying resistance to traditional antiplatelet drugs has been described (Stef, Csiszar, Ziangmin, Ferdinandy, Ungvari \& Veress, 2007), suggesting that targeting NOXs may become a valid alternative antithrombotic strategy (Violi \& Pignatelli, 2014).

The work from our and other laboratories highlighted the central role of NOX1 in the signal transduction of collagen-dependent platelet activation (Vara \& Pula, 2014; Walsh, Berndt, Carrim, Cowman, Kenny \& Metharom, 2014). Recently, we utilized selective agonists and a novel electron paramagnetic resonancebased technique to show that the signalling of the collagen receptor GPVI requires NOX1 (Abubaker, Vara, Eggleston, Canobbio \& Pula, 2019). These results are paralleled by a recent study showing that NOX2 is dispensable for GPVI-dependent activation of platelets ex vivo, and the carotid thrombosis induced by photochemical injury in vivo (Sonkar et al., 2019). This recent evidence is in contrast with previous studies indicating NOX2 as critical for the activation of platelets by collagen and for the development of thrombosis in vivo (Delaney et al., 2016). Although differences in the methodologies may partly explain this discrepancy (e.g. ferric chloride vs photochemical vascular injuryin vivo ), the exact roles of different NOX family members in the redox-dependent regulation of platelets remain to be fully explained. Other aspects that remain to be elucidated are the mechanism of activation of NOXs in platelets and the downstream signalling that they activate. Currently, a role for the small GTPase Rac1 has been proposed for the activation of NOXs in platelets (Akbar et al., 2018; Akbar, Duan, Saleem, Davis \& Zheng, 2016), while the most likely mechanism of signal transduction that they triggers involves the oxidative inaction of the protein phosphatase SHP2 resulting in potentiation of the protein kinase cascade activated by GPVI in response to collagen (Jang et al., 2015; Wang et al., 2015).

In this study, using NOX1 ${ }^{-/}$platelets, we firstly confirmed the role of NOX1 and NOX2 in platelet aggregation induced by collagen and thrombin ex vivo. This indicated NOX1 as the critical NOX family member for collagen-induced platelet activation but not thrombin-dependent aggregation. We then utilized a ferric chloride-induced carotid occlusion model to study the effect of NOX1- or NOX2-deficiency on atherothrombosis (Grover \& Mackman, 2019). NOX1-deficiency resulted in a significant increase in the time necessary to reach carotid occlusion. In parallel, neither NOX1 nor NOX2 deficiency affected the hemostatic response in a tail tip amputation assay. These results suggested that the inhibition of NOX1 may represent a viable pharmacological strategy for the treatment or prevention of thrombotic conditions without impairing hemostatic responses. We therefore tested the established NOX1 inhibitor 2-acetylphenothiazine (2APT) and nine chemical derivatives (2APT-D1/9). 2APT-D6 was selected as the most potent derivative in ex vivo platelet tests and was further characterized. Using $\mathrm{NOX}^{-/-}$platelets, we confirmed the selectivity of $2 \mathrm{APT}$ and 2APT-D6 for NOX1. Ex vivoexperiments suggested that 2APT-D6 is threefold more potent than the parent molecule 2APT. Nonetheless, when administered orally in mice, both 2APT and 2APT-D6 elicited a comparable level of protection against carotid occlusion without impairing hemostasis. This study characterizes two NOX1-selective inhibitors with the potential to become novel orally-deliverable drugs. The ability of 2APT and 2APT-D6 to selectively impair collagen-induced platelet activation and protect against thrombosis without affecting hemostasis in vivo are particularly important advantages compared to existing drugs.

\section{METHODS}

\section{APT derivative synthesis}

Detailed synthesis and purity control procedures are described in the Methods Supplement. Structures are described in Table 1.

Mouse maintenance and pharmacological treatment 


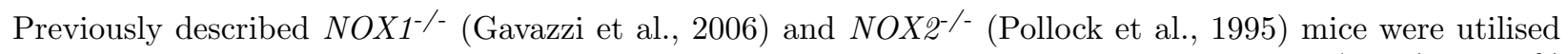
for this study. Drug administration was obtained by mixing either 2 APT or 2 APT-D6 $(2 \mathrm{mg} / \mathrm{ml}$ in $2 \%$ $\mathrm{v} / \mathrm{v}$ DMSO) with solid food to reach a dose of $400 \mathrm{mg}$ of medication per $\mathrm{kg}$ of mouse body weight (200 $\mathrm{mg} / \mathrm{kg}$ ). $2 \% \mathrm{v} / \mathrm{v}$ DMSO vehicle solution was used as a negative control. Allin vivo studies were performed according to local ethical approval (University of Exeter) and Home Office licencing for Scientific Procedures (PPL30/3348).

\section{Murine blood collection and platelet preparation}

Blood was collected from 12 weeks old female mice by cardiac puncture. For washed platelets, sodium citrate was used as an anticoagulant $(0.5 \% \mathrm{w} / \mathrm{v})$. Platelet rich plasma (PRP) was separated from whole blood by centrifugation $(180 \times \mathrm{g}, 15$ minutes $)$, and platelets were separated from PRP by a second centrifugation step $(600 \times \mathrm{g}, 10$ minutes), in the presence of prostaglandin E1 (PGE1, $40 \mathrm{ng} / \mathrm{ml})$ and indomethacin (10 $\mu \mathrm{M})$. Platelets were resuspended in modified Tyrode's buffer at a density of $2 \times 10^{8}$ platelets $/ \mathrm{ml}$ throughout the study. For whole blood analyses (e.g. thrombus formation), heparin- and PPACK-anticoagulated whole blood was used ( 5 unit $/ \mathrm{ml}$ and $25 \mu \mathrm{M}$, respectively).

\section{Human blood collection and platelet preparation}

Human blood was drawn from healthy volunteers by median cubital vein venepuncture following Royal Devon and Exeter NHS Foundation Trust Code of Ethics and Research Conduct and under National Research Ethics Service South West - Central Bristol approval (Rec. n. 14/SW/1089). Sodium citrate was used as anticoagulant $(0.5 \% \mathrm{w} / \mathrm{v})$. Platelet rich plasma (PRP) was separated from whole blood by centrifugation [250xg, 17 minutes (min)], and platelets were separated from PRP by a second centrifugation step (500xg, $10 \mathrm{~min}$ ), in the presence of prostaglandin E1 (PGE1, $40 \mathrm{ng} / \mathrm{mL}$ ) and indomethacin $(10 \mu \mathrm{M})$. For whole blood analyses (e.g. thrombus formation), heparin- and PPACK-anticoagulated whole blood was used.

\section{Platelet aggregation by turbidimetry}

Platelets resuspended in modified Tyrode's buffer at a density of $2 \times 10^{8}$ platelets $/ \mathrm{ml}$ were stimulated using a Chrono-Log $4904+$ aggregometer (Havertown, US). Aggregation was induced with $0.1 \mathrm{unit} / \mathrm{ml}$ human thrombin or $3 \mu \mathrm{g} / \mathrm{ml}$ Horm collagen. Absorbance was measured for 10 minutes and expressed as $\%$ change of absorbance.

\section{Carotid occlusion model}

The procedure has been performed as previously published (Bonnard \& Hagemeyer, 2015) and is described in the Methods Supplement. Briefly, the carotid artery of anaesthetized mice was surgically exposed and fitted with a miniature transonic flow probe. Ferric chloride $\left(\mathrm{FeCl}_{3}, 10 \% \mathrm{w} / \mathrm{v}\right)$ was applied topically for 5 minutes, and flow was continuously recorded until cessation (i.e. occlusion) or alternatively up to 40 minutes in occlusion-free mice.

\section{Tail tip transection assay}

Mice were anesthetized by inhalation of $4 \%$ isoflurane and continuously maintained at $1.5 \%$ isoflurane. The animals were placed in prone position and a $3-\mathrm{mm}$ segment of the tail was amputated with a scalpel. The tail was immediately immersed in pre-warmed isotonic saline at $37{ }^{\circ} \mathrm{C}$. Bleeding time was measured manually.

\section{Electron paramagnetic resonance}

As described in our previous study (Abubaker, Vara, Eggleston, Canobbio \& Pula, 2019). Prior to adding stimuli, $200 \mu \mathrm{M}$ 1-hydroxy-3-methoxycarbonyl-2,2,5,5-tetramethylpyrrolidine (CMH), $5 \mu \mathrm{M}$ diethyldithiocarbamate (DETC) and $25 \mu \mathrm{M}$ deferoxamine were added to platelets (density adjusted to $2 \times 10^{8}$ platelets $/ \mathrm{ml}$ ) with continuous stirring. After 1 minute, stimuli were delivered, and 10 minutes later $50 \mu$ of platelet-free supernatant were collected and read using an E-scan (Noxygen, Germany). EPR spectra were recorded using the following EPR settings: centre field 3,490 G, field sweep $60 \mathrm{G}$, modulation amplitude $2 \mathrm{G}$, sweep time $10 \mathrm{~s}$, number of scans 10, microwave frequency $9.39 \mathrm{GHz}$. A calibration curve was obtained from standard 
$\mathrm{CM}^{*}$ diluted to concentrations of $0,0.3,1,3,10$, and $30 \mu \mathrm{M}$ and utilized to estimate the $\mathrm{CM}^{*}$ concentration in the samples as described in Supplementary Figure 1. The CMH oxidation rate was obtained using the following formula: $\mathrm{CMH}$ oxidation rate $=\left[\mathrm{CM}^{*}\right] \mathrm{x}$ Volume / (Platelet density $\mathrm{x}$ Volume $\mathrm{x}$ Time)

\section{Platelet static adhesion}

Platelets at a density of $2 \times 10^{7}$ platelets $/ \mathrm{ml}$ were treated with NOX inhibitors for 10 minutes and then seeded on collagen- or fibrinogen-coated coverslips for $30 \mathrm{~min}$ at $37^{\circ} \mathrm{C}$. After removal of non-adhering cells, platelets were fixed with $4 \%$ paraformaldehyde for $10 \mathrm{~min}$. After permeabilization with $0.1 \%$ Triton X/PBS, $10 \mu \mathrm{M}$ TRITC-conjugated phalloidin was utilized to stain the actin cytoskeleton. Quantification of platelet adhesion was obtained by LED fluorescence microscopy (EVOS Fl) and image analysis using Image J 1.47v (Wayne Rasband, National Institute of Health, USA).

\section{Thrombus formation under physiological flow assay}

Human and mouse blood was anticoagulated with $5 \mathrm{u} / \mathrm{ml}$ heparin and $40 \mu \mathrm{M}$ D-Phenylalanyl-prolyl-arginyl Chloromethyl Ketone (PPACK), and labelled with $1 \mu \mathrm{M}$ 3,3'-Dihexyloxacarbocyanine Iodide (DiOC6) for 10 minutes. Ibidi Vena8 Fluoro+ flow microchips and a Cellix Exigo pump were utilized to analyze thrombus formation in whole blood under flow. Microchips were coated with Horm collagen $(0.1 \mu \mathrm{g} / \mathrm{ml})$. Thrombus formation was visualized by fluorescence microscopy at a shear rate of $1,000 \mathrm{sec}^{-1}$. Surface coverage was analyzed using ImageJ 1.47v (Wayne Rasband, National Institute of Health, USA).

\section{Flow cytometry}

As previously described (Rochat \& Alberio, 2015), washed human platelets were stimulated with $1 \mathrm{unit} / \mathrm{ml}$ thrombin or $5 \mu \mathrm{g} / \mathrm{ml}$ cross-linked collagen-related peptide (CRP-XL) and fixed in 1\% w/v paraformaldehyde for 30 minutes. After diluting 1 in 10 in modified Tyrode's buffer, FITC-conjugated anti-activated integrin

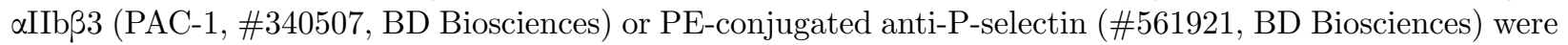
used to stain platelets and fluorescence staining was assessed using a FACS Aria III flow cytometer (BD Biosciences).

\section{Carotid occlusion model}

The procedure has been performed as described previously described (Bonnard \& Hagemeyer, 2015). Briefly, anaesthesia was induced with $5 \%$ isoflurane and maintained during spontaneous breathing of isoflurane at $2 \%$ with oxygen. The carotid artery was surgically exposed and fitted with a miniature transonic flow probe (Transonic Systems Inc. TS420, USA). The probe was linked to a CED Micro1401 acquisition unit (Cambridge Electronic Design Limited, UK) via a flowmeter (Transonic Systems Inc., USA). Ferric chloride $\left(\mathrm{FeCl}_{3}, 10 \% \mathrm{w} / \mathrm{v}\right)$ was applied topically to the exposed artery via a piece of filter paper $(1 \mathrm{x} 1.5 \mathrm{~mm})$ to induce thrombus formation. After 5 minutes, the filter paper was removed while blood flow was continuously recorded until cessation (i.e. occlusion) or alternatively up to 40 minutes in occlusion-free mice. Data were acquired using Spike2 (Cambridge Electronic Design Limited, UK) software. Time to occlusion after FeCl3 challenge was analyzed and utilized as a measure of susceptibility to thrombosis. Hematoxylin staining of explanted carotid sections after ferric chloride treatment was used to confirm thrombus formation.

\section{Statistical Analysis}

For dual comparisons of normal/homoscedastic data, statistical analysis was performed by one-tailed unpaired t-tests. Dual comparisons (i.e. WT vs 3KO) of non-normal/non-homoscedastic data were analyzed by one tailed non-parametric Mann-Whitney test. One-way ANOVA with Bonferroni post-test was used for multiple comparison tests of normal/homoscedastic data. Data normality and homoscedasticity were tested with Shapiro-Wilk and Bartlett's tests, respectively. Static adhesion experiments were normalized and analysed by one sample t-test versus $100 \%$ for the untreated condition. The statistical software package GraphPad Prism Version 8.1.0 for Windows 64 bit was used. Results were expressed as the mean \pm standard error (SEM). Differences were considered significant at $P$ value $<0.05\left(^{*}\right), 0.01(* *)$ or $0.001(* * *)$.

\section{Results}


In order to clarify the differential role of NOX1 and NOX2 in the regulation of platelets, we analyzed thrombin- and collagen-induced aggregation of platelets from $\mathrm{NOX}^{-/-}, \mathrm{NOX}^{-/-}$and wild type mice $(\mathrm{C} 57 \mathrm{Bl6} / \mathrm{J})$. As shown in in Figure $1 \mathrm{~A}$, collagen-induced aggregation is significantly inhibited in NOX1-/, while thrombininduced aggregation is significantly reduced in NOX2 $2^{-/-}$platelets (quantification in Supplementary Figure 2A). Next, in order to characterize the role of NOX1 and NOX2 in thrombotic and haemostatic responses in vivo, we characterized carotid occlusion (Figure 1B, and representative traces in Supplementary Figure 3) and tail tip transection bleeding time (Figure 1C), in $\mathrm{NOX}^{-/-}, \mathrm{NOX}^{-/-}$and wild type mice. This analysis showed that $\mathrm{NOX}^{-/-}$mice have significantly delayed carotid occlusion compared to wild type mice, while tail haemostasis is not affected. NOX2-/- appear normal for both thrombotic and haemostatic responses.

Based on the importance of NOX1 in atherothrombosis in vivo, we focused the rest of this study on the antithrombotic effects of novel derivatives of a known inhibitor of this enzyme: 2-acetylphonothiazine (2APT) (Gianni et al., 2010). Firstly, we analyzed the effect of 2APT on the oxidative response induced by collagen and thrombin in wild type and $\mathrm{NOX}^{-/-}$platelets. We utilized an electron paramagnetic resonance (EPR) spectroscopy method that we adapted and validated for platelets that allows the measurement of superoxide anion output during stimulation (Abubaker, Vara, Eggleston, Canobbio \& Pula, 2019). The calibration curve utilized to quantify the superoxide anion output is shown in Supplementary Figure 1. As expected for a NOX1-selective inhibitor, 2APT significantly inhibits the superoxide anion generation induced by collagen on wild type platelets, but not in NOX1/- platelets (Figure $2 \mathrm{~A}$ ). The superoxide anion generation in response to thrombin is also inhibited by $2 \mathrm{APT}$, although $1 \mu \mathrm{M}$ is significantly less effective than $10 \mu \mathrm{M}(\sim 25 \%$ inhibition vs $\sim 80 \%$, Figure $2 \mathrm{~B}$ ). This may confirm a previously-reported non-selective effect of $2 \mathrm{APT}$ on NOX2 only at high concentrations $(>5 \mu \mathrm{M})$ (Gianni et al., 2010). In accordance with this, the inhibitory effect at $10 \mu \mathrm{M}$ $2 \mathrm{APT}$ is also detectable in NOX1 ${ }^{-/-}$platelets stimulated by thrombin (Figure 2B). The effect of $2 \mathrm{APT}$ on collagen- and thrombin-dependent aggregation of wild type and $\mathrm{NOX}^{-/-}$platelets was then tested. $2 \mathrm{APT}$ inhibits collagen-dependent aggregation of wild type platelets in concentration-dependent manner $\left(\mathrm{IC}_{50}=\right.$ $141 \pm 42 \mathrm{nM}$, Figure 2Ci), while the effect in NOX1 ${ }^{-/-}$is apparent only at $10 \mu \mathrm{M}$ (Figure 2Cii). On the other hand, thrombin-dependent aggregation in wild type and $\mathrm{NOX}^{-/-}$platelets is only marginally affected by 2APT and only at the highest concentration $(10 \mu \mathrm{M})$ (Figures 2Di and 2Dii, quantification in Supplementary Figure $2 \mathrm{C})$.

In addition to $2 \mathrm{APT}$, a group of synthetic derivatives were designed, synthesized and examined in this study (2APT-D1/9). The compounds included structural modifications to the 2APT core (2APT-D2, $3 \& 7$ ), and two different prodrug approaches that could potentially release unmodified 2APT either upon hydrolysis then decarboxylation (2APT-D1, 4, 5, 8 \& 9), or simply upon hydrolysis directly (2APT-D6). The nine derivatives are shown in Table 1 and their syntheses described in the Methods Supplement. In order to identify compounds with antiplatelet properties amongst these derivatives, a static platelet adhesion assay was utilized. Platelet suspension were pre-treated with the compounds at $10 \mu \mathrm{M}$ concentration (Figure 3A) which allowed the selection of 2APT-D5, 2APT-D6, 2APT-D8 and 2APT-D9 for their ability to inhibit collagen adhesion to an extent similar or superior to 2APT. It is interesting to note that the compounds retaining inhibitory activity are all potential prodrugs of $2 \mathrm{APT}$, while the modifications of the $2 \mathrm{APT}$ core abolished the biological activity of the molecule. Further analysis of these compounds at concentrations of 1 and $0.1 \mu \mathrm{M}$ suggested that 2APT-D6 is the most potent derivative of 2APT (Supplementary Figure 4). In parallel, neither 2APT nor its derivatives inhibit platelet adhesion to fibrinogen (Figure 3B). These compounds were also tested by platelet aggregation $(3 \mu \mathrm{M})$, where similarly to adhesion experiments, only 2APT, 2APT-D5, 2APT-D6, 2APT-D8 and 2APT-D9 inhibited collagen responses, while thrombin was not affected (Figure 3C and 3E).

Based on the above experiments, 2APT-D6 was selected and further characterized. Similarly to 2APT: 1) 2APT-D6 significantly inhibits superoxide anion formation in response to collagen at similar extent at 1 or $10 \mu \mathrm{M}$ in wild type mouse platelets (Figure 4A); 2) NOX1 specificity cannot be tested in $\mathrm{NOX}^{-/-}$platelet stimulated by collagen as the agonist-induced superoxide generation is absent (Figure 4A); 3) the inhibition of superoxide generation in response to thrombin in is significantly higher for $10 \mu \mathrm{M}$ than $1 \mu \mathrm{M} 2 \mathrm{APT}-\mathrm{D} 6$ in wild type mouse platelets ( $85 \%$ inhibition vs $60 \%$, Figure $4 \mathrm{~B}$ ); 4) only $10 \mu \mathrm{M}$ but not $1 \mu \mathrm{M} 2 \mathrm{APT}-\mathrm{D} 6$ 
has some inhibitory effect on thrombin-dependent superoxide generation in NOX1-/- (Figure 4B). Tested at different concentrations in aggregation assays, 2APT-D6 inhibits collagen-dependent aggregation with higher potency than 2-APT $\left(\mathrm{IC}_{50}=52 \pm 18 \mathrm{nM}\right.$, Figure $4 \mathrm{Ci}$ ). In order to verify the selectivity of 2APT-D6 for NOX1, NOX1 ${ }^{-/-}$platelets were tested by turbidimetry and resulted insensitive to 2APT-D6 except for the highest concentration of $10 \mu \mathrm{M}$ (Figure 4Cii). In order to prove the selectivity of 2APT-D6 for the signalling of the collagen receptor GPVI, the selective agonist cross-linked collagen related peptide (CRP-XL) was used (Jarvis et al., 2008) (Figure 4D). 2APT-D6 inhibited platelet aggregation induced by $1 \mu \mathrm{g} / \mathrm{ml} \mathrm{CRP-XL} \mathrm{in} \mathrm{a}$ concentration-dependent manner (Figure 4Di and 4Diii), with $\mathrm{IC}_{50}$ of $77 \pm 24 \mathrm{nM}$ (Figure 4Diii). Similarly, 2APT inhibited CRP-XL-dependent platelet aggregation in a concentration-dependent manner with $\mathrm{IC}_{50}$ of $179 \pm 37 \mathrm{nM}$ (Figure 4Dii and 4Diii).

Furthermore, 2APT and 2APT-D6 were compared for their ability to inhibit thrombus formation in a whole blood flow assay (Nagy et al., 2019). Data in Figure 5A show a comprehensive inhibition of thrombus formation by either compounds at $10 \mu \mathrm{M}$, but a significantly higher inhibition of thrombus formation for 2APT-D6 at $1 \mu \mathrm{M}$ compared to 2APT. Next, we analysed the effect of 2APT and 2APT-D6 on CRP-XLand thrombin-dependent platelet activation by flow cytometry. Integrin $\alpha \operatorname{IIb} \beta 3$ activation (Figure 5Bii) and P-selectin externalization (i.e. degranulation) (Figure 5Biii) were analyzed. Both 2APT (red bars) and 2APT-D6 (blue bars) at concentrations of $0.1 \mu \mathrm{M}$ or more significantly inhibited $\alpha \mathrm{IIb} \beta 3$ activation by $3 \mu \mathrm{g} / \mathrm{ml}$ CRP-XL (Figure 5Ci, green bar). Notably, 2APT-D6 appeared significantly more potent at $0.1 \mu \mathrm{M}$ than $2 \mathrm{APT}$ (red connector lines and asterisks). The activation of $\alpha \operatorname{IIb} \beta 3$ in response to $1 \mathrm{unit} / \mathrm{ml}$ thrombin was significantly inhibited only by the highest tested concentration for both compounds (i.e. $10 \mu \mathrm{M}$, Figure 5Cii). In parallel, P-selectin externalization induced by $3 \mu \mathrm{g} / \mathrm{ml} \mathrm{CRP-XL} \mathrm{(green} \mathrm{bar)} \mathrm{was} \mathrm{significantly} \mathrm{inhibited} \mathrm{by}$ 2APT (red bars) and 2APT-D6 (blue bars) at concentrations of $0.1 \mu \mathrm{M}$ or more (Figure 5Di), with 2APT-D6 significantly more potent than $2 \mathrm{APT}$ at $0.1 \mu \mathrm{M}$ (red connector lines and asterisks). P-selectin externalization induced by $1 \mathrm{unit} / \mathrm{ml}$ thrombin was significantly inhibited only by the highest tested concentration of either compound (i.e. $10 \mu \mathrm{M}$, Figure 5Dii).

Both 2APT and 2APT-D6 were then tested in vivo . The compounds were administered in the diet by mixing ground chow diet pellet with $2 \mathrm{mg} / \mathrm{ml}$ solution of the compounds (in $2 \% \mathrm{v} / \mathrm{v}$ dimethyl sulfoxide) to obtain a daily dose of $200 \mathrm{mg} / \mathrm{kg} .2 \% \mathrm{v} / \mathrm{v}$ dimethyl sulfoxide was added to the food as negative control (i.e. vehicle). After two full days of treatment homogeneates of liver, kidney and platelet-rich plasma were analyzed by liquid chromatography - mass spectrometry (LC-MS) for the presence of 2APT (Supplementary Figure 5). 2APT was detected in liver, kidney and plasma homogeneates of animals treated with 2APT. Interestingly, 2APT also appeared in the samples from animals treated with 2APT-D6, therefore proving that this molecule acts as a prodrug able to release 2APT. mice were tested as described. Washed platelets were tested by EPR, which showed significant inhibition of collagen-dependent superoxide generation (Figure 8Aii, and quantification in Figure 8Ai), while the thrombin-dependent superoxide output in response to thrombin was not affected (Figure 6Aiii, and quantification in Figure 6Ai). In parallel, washed platelet turbidimetry was utilized and both compounds comprehensively inhibited collagen-dependent aggregation (Figure 6Bi, and quantification in Supplementary Figure $2 \mathrm{~F}$ ), but had no effect on thrombin-dependent aggregation (Figure 6Bii, and quantification in Supplementary Figure 2G).

Whole blood from the mice treated as described above was tested for thrombus formation on collagen under physiological flow $\left(1,000 \mathrm{sec}^{-1}\right)$. Treatment with either 2APT or 2APT-D6 comprehensively abolished thrombus formation (representative examples in Figure 7Ai and quantification in Figure 7Aii). The induction of carotid occlusion by ferric chloride treatment was also significantly delayed in 2APT- and 2APT-D6-treated animals (Figure 7Bi shows mean occlusion time \pm SEM for the different conditions, while Figure 7Bii shows hematoxylin staining of treated carotids). Notably, haemostasis as tested in tail tip transection bleeding time assays was not affected by treatment with either 2APT or 2APT-D6 (Figure 7C).

\section{Discussion}

Using NOX1 ${ }^{-/-}$and NOX2 ${ }^{-/-}$transgenic mice (Gavazzi et al., 2006; Pollock et al., 1995) and a model of carotid occlusion induced by ferric chloride (Bonnard \& Hagemeyer, 2015), we proved that NOX1 is required 
for thrombotic responses. In parallel, haemostasis tested in tail tip transection assays (Vaezzadeh, Ni, Kim, Weitz \& Gross, 2014) is unaffected by NOX1 deficiency. NOX1 is therefore a promising target for the development of pharmacological agents able to protect against thrombosis without increasing bleeding risk. Using washed platelets from $\mathrm{NOX}^{-/-}$and $\mathrm{NOX}^{-/-}$transgenic mice we have also proved that NOX1 is critical for collagen responses, while the latter is required for thrombin responses. This is in agreement with previous studies (Abubaker, Vara, Eggleston, Canobbio \& Pula, 2019; Vara, Campanella \& Pula, 2013; Walsh, Berndt, Carrim, Cowman, Kenny \& Metharom, 2014), but in contrast with other reports (Cammisotto et al., 2019; Delaney et al., 2016). This discrepancy is difficult to fully understand, although it can be at least partially explained with the differences in experimental tools and conditions utilized. For example, the inhibitor peptide used as NOX2-specific by Cammisotto and colleagues (Cammisotto et al., 2019) directly binds and competitively inhibits p47phox (Csanyi et al., 2011). Therefore, in addition to inhibiting NOX2, this peptide can potentially inhibit p47phox-dependent activation of other NOXs (including NOX1 (Bedard \& Krause, 2007; Rezende et al., 2018; Youn, Gao \& Cai, 2012)) and the results from this manuscript may be reinterpreted under a different light. Interestingly, our conclusions on the role of NOX1 in collagen signalling are in agreement with a recent paper showing no changes in platelet responses to collagen in NOX2 $2^{-/}$mice and Cybb (NOX2)-mutation bearing granulomatous disease (CGD) patients (Sonkar et al., 2019).

Previous data suggested a role for NOX1 in the regulation of the collagen receptor GPVI (Abubaker, Vara, Eggleston, Canobbio \& Pula, 2019; Vara, Campanella \& Pula, 2013; Walsh, Berndt, Carrim, Cowman, Kenny \& Metharom, 2014). Accordingly, genetic silencing or pharmacological inhibition of GPVI has been shown to reduce thrombotic responses without affecting haemostasis in a manner similar to what we observed for NOX1-/- (Arthur, Dunkley \& Andrews, 2007; Jiang \& Jandrot-Perrus, 2014). Unsurprisingly, GPVI targeting has previously been proposed as a potential strategy for the prevention of atherothrombosis (Jiang \& Jandrot-Perrus, 2014; Nieswandt et al., 2001). Because only peptides or antibodies have so far been successfully utilized as pharmacological agents targeting GPVI (Florian, Wonerow, Harder, Kuczka, Dubar \& Graff, 2017; Massberg et al., 2004; Mojica Munoz et al., 2017; Voors-Pette et al., 2019), GPVI remains an interesting drug discovery target. In this study we investigated the possibility of targeting NOX1 and indirectly dampening GPVI signalling with small molecule inhibitors. A number of small molecule NOX inhibitors with isotype selectivity have recently been developed (Gianni et al., 2010; Gray et al., 2017; Hirano et al., 2015; Teixeira et al., 2017). We previously described the effect of the NOX1-selective inhibitor 2APT on platelets ex vivo (Vara, Campanella \& Pula, 2013). Here we further characterized the antiplatelet potential of $2 \mathrm{APT}$ both ex vivo and in vivo. In addition, in order to explore the effects of structural changes on the potency and selectivity of this inhibitor, we designed, synthesized and examined nine related chemical derivatives of $2 \mathrm{APT}$. The $2 \mathrm{APT}$ derivatives $2,3 \& 7$ possess modifications to the $2 \mathrm{APT}$ structure, however, the remaining derivatives all have the potential to release unmodified 2APT, via two different pathways. Specifically, derivatives 2APT-D1, 4, 5, 8 \& 9 can undergo hydrolysis to afford a common $\beta$-keto acid intermediate that can then undergo enzymatic decarboxylation to release 2APT (Bach \& Canepa, 1996). In an alternative approach, 2APT-D6 contains a vinyltert -butyl carbonate that upon hydrolysis is able to afford 2APTdirectly. A similar prodrug approach using a tert -butyl carbonate motif was examined with orally administered entacapone and was reported to be quantitatively hydrolysed to the drug in serum (Leppanen et al., 2001).

In our experiments, a number of 2APT derivatives lost their ability to affect platelet activation (2APTD1, 2APT-D2, 2APT-D3, 2APT-D4 and 2APT-D7), but amongst inhibitors retaining antiplatelet activity, 2APT-D6 displayed higher potency than 2APT in aggregation experiments $\left(\mathrm{IC}_{50}=52 \pm 18 \mathrm{nM}\right.$ vs $141 \pm$ $42 \mathrm{nM}$ with collagen as agonist, and $77 \pm 24 \mathrm{nM}$ vs $179 \pm 37 \mathrm{nM}$ with CRP-XL as agonist). These results demonstrate that changes to the structure of $2 \mathrm{APT}$ are not tolerated (2APT-D2, $3 \& 7$ ) and that the methyl and ethyl $\beta$-ketoesters are not effective substrates (2APT-D1 \& 4, respectively). However, derivative 2APT-D6, which hydrolyses to afford 2APT directly, showed the most promise. EPR spectroscopy and platelet aggregation in combination with the use of $\mathrm{NOX1}^{-/}$platelets confirmed the selectivity of $2 \mathrm{APT}$ and 2APT-D6 for NOX1. This became apparent in experiments using NOX1-/-platelets, where only the highest concentration of inhibitors had an effect (i.e. $10 \mu \mathrm{M}$, Figure $2 \mathrm{~A}$ and $2 \mathrm{C}$ ). At this high concentration the 
inhibitors are likely to affect NOX2, which has been shown in a previous study $\left(\mathrm{IC}_{50}\right.$ for $2 \mathrm{APT}$ in NOX2expressing cells $=5 \mu \mathrm{M})$ (Gianni et al., 2010). Interestingly, the inhibition of aggregation and superoxide anion formation in NOX1 $1^{-/}$is in line with our recent publication showing that collagen activates preferentially NOX1 (Abubaker, Vara, Eggleston, Canobbio \& Pula, 2019). In fact, EPR detection of superoxide anion generation (Figure 2A) and aggregation (Figure 2C) in response to collagen are strongly inhibited in $\mathrm{NOX}^{-/}$platelets compared to wild type platelets. The situation is different for thrombin, which appears to activate both NOX1 and NOX2 (although the NOX2 component seems more prominent). NOX1/-- platelets have significantly reduced superoxide anion output (Figure 2B) and aggregation (Figure 2C) in response to thrombin, but a sizeable response remains in either assays. Again, 2APT and 2APT-D6 only affected thrombin responses in $\mathrm{NOX}^{-/-}$platelets at a concentration of $10 \mu \mathrm{M}$, which, as mentioned above, has been shown to inhibit NOX2 (Gianni et al., 2010).

In addition to characterizing their potency and selectivity ex vivo, we tested 2APT and 2APT-D6 in vivo - This allowed to investigate their effect on complex physiological phenomena, such thrombus formation and haemostasis, but it also provided valuable information on safety and bioavailability of these compounds. It was very promising to see that $2 \mathrm{APT}$ and 2APT-D6 orally administered to mice significantly reduced platelets responses (i.e. aggregation, superoxide anion formation and thrombus formation under flow Figure 6). This suggests that the compounds are not degraded in the GI tract, are absorbed and reach the blood at concentrations compatible with their biological activity. 2APT could in fact be detected by mass spectrometry in kidney, liver and platelet-rich plasma lysates of treated animals but not control animals that received vehicle solution (Supplementary Figure 5). The prodrug mechanism of 2APT-D6 was confirmed by mass spectrometry experiments showing the parent molecule 2APT in animals treated with 2APT-D6 (Supplementary Figure 5C). A novel molecule appeared in the plasma of animals treated with 2APT and 2APT-D6, which was identified as a 2APT-glucose adduct. Further studies on the relevance of this molecule for absorption and biological activity of 2APT and 2APT-D6 are required. The increased potency of 2APTD6 over $2 \mathrm{APT}$ that we observed ex vivo could not be observed in vivo, possibly because of the conversion of the former molecule into the latter in vivo. The oral bioavailability associated with the proved in vivo efficacy of 2APT and 2APT-D6 make these molecules promising candidates for drug discovery and give them a potential advantage over existing GPVI inhibitors (Florian, Wonerow, Harder, Kuczka, Dubar \& Graff, 2017; Massberg et al., 2004; Mojica Munoz et al., 2017; Voors-Pette et al., 2019), which display the cost and pharmacokinetic limitations of peptide- and antibody-based drugs. Our in vivo studies confirmed the potential of 2APT and 2APT-D6 by showing a decisive increase in the carotid occlusion time. This assay is commonly used to assess thrombosisin vivo and is based on the use of ferric chloride $\left(\mathrm{FeCl}_{3}\right)$ to induce vascular endothelial cell damage (Strassel et al., 2007). Promisingly, the administration of these drugs did not affect bleeding time in tail tip transection assays. This suggests that coagulation and non-collagen-dependent platelet activation are sufficient to ensure normal haemostasis in the presence of 2APT and 2APT-D6.

In summary, 2APT and 2APT-D6 selectively inhibit collagen-dependent activation of platelets without affecting thrombin responses (Figure 8), which reduces thrombosis without attenuating haemostasis. These compounds could therefore be developed into antithrombotic drugs without the unwanted side effect of increasing bleeding risks (Buresly, Eisenberg, Zhang \& Pilote, 2005; Swan, Loughran, Makris \& Thachil, 2019; Yasuda et al., 2015), which is an unresolved weakness of existing antiplatelet drugs.

\section{Acknowledgement}

This work was funded by a British Heart Foundation grant (PG/15/40/31522) and Alzheimer Research UK (ARUK-PG2017A-3) to Giordano Pula. Work in Maarten Koeners' laboratory was supported by the British Heart Foundation (FS/14/2/30630 and PG/15/68/31717). The authors would also like to thank the Biological Service Unit of the University of Exeter for the in vivo work, the Clinical Research Facility (CRF) of the University of Exeter and in particular Dr Bridget Knight for blood collections, the Chemical Characterisation and Analysis Facility (CCAF) of the University of Bath (especially Dr Anneke Lubben and Dr Shaun Reeksting) for the mass spectrometry analysis, and Dr Bruno Fink from Noxygen Science Transfer \& Diagnostics GmbH for the technical support. 


\section{Author contribution}

DV performed and analyzed the majority of the experiments, with help forin vivo treatment (AT), platelet adhesion (MC), and carotid occlusion assays (DP, BF and ZW). MPK supported the carotid occlusion experiments and participated in the preparation of the manuscript. CEG and EH performed the synthesis of the novel compounds tested. LC designed and synthesized the compounds, and participated in the writing of the manuscript. GP designed the study, generated part of the data, analyzed all of the data, and wrote the manuscript.

\section{Conflict of interests}

The authors declare no conflict of interest.

\section{Figure legends}

\section{Table 1: 2APT derivative structures.}

Figure 1: NOX1 positively regulates collagen-dependent activation of platelets and facilitates thrombosis in vivo.Platelets were isolated from $\mathrm{NOX}^{-/-}$, NOX2 ${ }^{-/-}$and wild type mice (WT, C57BL6/J) and resuspended at $2 \times 10^{8}$ cells $/ \mathrm{ml}$. Aggregation was obtained with $3 \mu \mathrm{g} / \mathrm{ml}$ collagen (Ai) or $0.1 \mathrm{unit} / \mathrm{ml}$ thrombin (Aii) and measured for up to 10 minutes. Traces shown in this figure are representative of 4 independent experiments (quantitative analysis is shown in Supplementary Figure 2A). NOX1-/-, NOX2-/and WT mice were also tested in a ferric chloride-induced carotid occlusion thrombosis assay. The time required for complete occlusion from 6 animals is displayed in (Bi), while hematoxylin staining of explanted carotid sections after ferric chloride treatment is shown in Bii. The haemostatic response was assessed with a tail tip transection assay and the time necessary for complete interruption of the bleeding from 9 animals per genotype is shown in (C). Statistical analysis in $\mathrm{B}$ and $\mathrm{C}$ was performed by one-way ANOVA with Bonferroni post-test ( $\mathrm{n}=6$ and 9 , respectively, and ${ }^{*}=\mathrm{p}<0.05$ ).

Figure 2: 2APT selectively inhibits NOX1 in superoxide anion formation and aggregation assays. Platelets were isolated from $\mathrm{NOX}^{-/-}$and WT mice $(\mathrm{C} 57 \mathrm{BL} 6 / \mathrm{J})$ and resuspended at $2 \times 10^{8}$ cells $/ \mathrm{ml}$. Superoxide anion output was measured by EPR following platelet activation with $3 \mu \mathrm{g} / \mathrm{ml}$ collagen (A) or $0.1 \mathrm{unit} / \mathrm{ml}$ thrombin (B) and measured for 10 minutes. Platelets were pretreated with 0,1 or 10 $\mu \mathrm{M} 2 \mathrm{APT}$ for 10 minutes. The amount of oxidised $\mathrm{CMH}$ was quantified as described in the Methods and in Supplementary Figure 1 and expressed as attomoles of CMH oxidised per platelet per minute. Data from 4 independent experiments $(\mathrm{n}=4)$ are shown in i) and representative EPR traces are shown in ii). Statistical analysis was performed by one-way ANOVA with Bonferroni post-test $\left(*\right.$ for $p<0.05$, ${ }^{* *}$ for $p<0.01,{ }^{* * *}$ for $\mathrm{p}<0.001$, ns for non-significant). $\mathrm{NOX}^{-/}$and WT platelets were also tested by turbidimetry following treatment with $2 \mathrm{APT}$ for 10 minutes $(0,0.03,0.1,0.3,0.3,1$ and 3 in $\mathrm{Ci}, 0,1$ and $10 \mu \mathrm{M}$ in Cii, Di and Dii). Platelet aggregation was stimulated with $3 \mu \mathrm{g} / \mathrm{ml}$ collagen (C) or $0.1 \mathrm{unit} / \mathrm{ml}$ thrombin (D). Representative examples from 4 independent are shown here (the quantification is shown in Supplementary Figure 2B and $2 \mathrm{C})$. A concentration-inhibition curve for $2 \mathrm{APT}$ on collagen calculated with data in $2 \mathrm{Ci}$ is shown in Figure $4 \mathrm{Ci}$.

Figure 3: 2APT derivatives inhibit static adhesion and aggregation of platelets in response to collagen. Human washed platelets were resuspended at $2 \times 10^{7}$ cells $/ \mathrm{ml}$ and incubated 10 minutes with $10 \mu \mathrm{M}$ 2APT, 2APT-D1, 2APT-D2, 2APT-D3, 2APT-D4, 2APT-D5, 2APT-D6, 2APT-D7, 2APT-D8 or 2APT-D9. Static adhesion to collagen (A) or fibrinogen (B) was tested by fluorescence microscopy and quantified. Representative micrographs are shown in i). The quantification of the results is shown in ii). Data were normalized to the control value in the absence of NOX1 inhibitors and expressed as \%. The statistical significance of the difference was tested by one-sample t-test $\left(\mathrm{n}=3,{ }^{*}\right.$ for $p<0.05,{ }^{* *}$ for $p<0.01,{ }^{* * *}$ for $\mathrm{p}<0.001$, ns for non-significant). The effect of $3 \mu \mathrm{M} 2 \mathrm{APT}$ derivatives on collagen- and thrombin-induced aggregation is shown in $\mathrm{C}$ and $\mathrm{D}$, respectively. Representative curves are shown in i), while statistical analysis is shown in ii) ( $\mathrm{n}=3$, one-way ANOVA with Bonferroni post-test, ${ }^{*}$ for $p<0.05$, ** for $p<0.01$, ${ }^{* * *}$ for $\mathrm{p}<0.001$, ns for non-significant). 
Figure 4: 2APT-D6 displays similar selectivity but higher potency than 2APT in superoxide anion formation and platelet aggregation assays. Platelets were isolated from $\mathrm{NOX}^{-/-}$and $\mathrm{WT}$ mice $(\mathrm{C} 57 \mathrm{BL} 6 / \mathrm{J})$ and resuspended at $2 \times 10^{8}$ cells $/ \mathrm{ml}$. Superoxide anion output was measured by EPR following platelet activation with $3 \mu \mathrm{g} / \mathrm{ml}$ collagen (A) or $0.1 \mathrm{unit} / \mathrm{ml}$ thrombin (B) and measured for 10 minutes. Platelets were pretreated with 0,1 or $10 \mu \mathrm{M} 2 \mathrm{APT}$ for 10 minutes. The amount of oxidised CMH was quantified as described in the Methods and in Supplementary Figure 1 and expressed as attomoles of $\mathrm{CMH}$ oxidised per platelet per minute. Data from 4 independent experiments $(\mathrm{n}=4)$ are shown in i) and representative EPR traces are shown in ii). Statistical analysis was performed by one-way ANOVA with Bonferroni post-test (* for $p<0.05,{ }^{* *}$ for $p<0.01,{ }^{* * *}$ for $\mathrm{p}<0.001$, ns for non-significant). WT (Ci) and NOX1 $^{-/-}$(Cii) platelets were also tested by turbidimetry following treatment with 2APT-6 for 10 minutes $(0$, $0.03,0.1,0.3,0.3,1,3$ or $10 \mu \mathrm{M})$. Platelet aggregation was stimulated with $3 \mu \mathrm{g} / \mathrm{ml}$ collagen. Concentrationinhibition curves for 2APT-D6 and 2APT (from Figure 2Ci) are also shown in Ci. Alternatively, 2APT-D6 (Di) and 2APT (Dii) were tested for their effect on WT platelet aggregation induced by $1 \mu \mathrm{g} / \mathrm{ml}$ CRPXL. Representative examples from 3 independent experiments are shown (the quantification is shown in Supplementary Figure 2D and E). In $\mathrm{Ci}$ and Diii, the aggregation values at 9 minutes were fitted to a variable slope sigmoidal concentration-response to obtain IC50 values (both results for 2APT and 2APT-D6 are shown (in red and green, respectively).

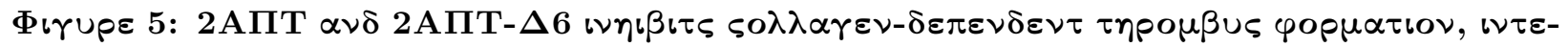

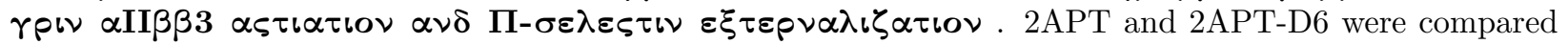
in whole blood thrombus formation assays under shear stress (A). The compounds $(1 \mu \mathrm{M})$ were incubated for 10 minutes on whole blood from human healthy volunteers where platelets were stained by DiOC6. The flow was applied for 10 minutes $\left(1,000 \mathrm{sec}^{-1}\right)$ before fluorescence pictures of the surfaces were taken to reveal the formation of thrombi. Representative examples are shown in $\mathrm{Ai}$, while statistical analysis is shown Aii $\left(\mathrm{n}=4\right.$, one-way ANOVA with Bonferroni post-test, ${ }^{*}$ for $p<0.05,{ }^{* *}$ for $p<0.01,{ }^{* * *}$ for $\mathrm{p}<0.001$, ns for non-significant). For flow cytometry, platelets were isolated from human blood, resuspended at $2 \mathrm{x}$ $10^{7}$ cells $/ \mathrm{ml}$ and activated with $3 \mu \mathrm{g} / \mathrm{ml}$ CRP-XL or $1 \mathrm{unit} / \mathrm{ml}$ thrombin in static conditions. The platelet population analyzed by flow cytometry is shown in $\mathrm{Bi}$ (forward scattering vs side scattering plot). Integrin $\alpha \operatorname{Ilb} \beta 3$ activation (Bii) and P-selectin externalization (Biii) were analysed by staining with activated $\alpha \operatorname{IIb} \beta 3$ (PAC-1) and P-selectin antibodies followed by flow cytometry. Platelets were incubated 10 minutes with 0 , $0.01,0.1,1$ or $10 \mu \mathrm{M}$ of $2 \mathrm{APT}$ or $2 \mathrm{APT}-\mathrm{D} 6$ before stimulation and immunostaining. The effect of $2 \mathrm{APT}$ and 2APT-D6 on integrin $\alpha \operatorname{IIb} \beta 3$ activation $(\mathrm{C})$ and P-selectin externalization (D) in response to CRP-XL (i) and thrombin (ii) is shown (mean $\pm \mathrm{SEM}, \mathrm{n}=4$ ). Statistical analysis was performed by one-way ANOVA with Bonferroni post-test, ${ }^{*}$ for $p<0.05,{ }^{* *}$ for $p<0.01,{ }^{* * *}$ for $\mathrm{p}<0.001$, ns for non-significant. Although results for 2APT and 2APT-D6 are similar, the effect of the compounds at concentration $0.1 \mu \mathrm{M}$ is statistically different (2APT-D6 causes more extensive inhibition, red lines and asterisk to indicate $\mathrm{p}<0.05)$.

Figure 6: In vivo administration of 2APT and 2APT-D6 selectively inhibits collagen-dependent superoxide anion formation and platelet aggregation. The drugs were administered in food (daily dose $=200 \mathrm{mg} / \mathrm{kg}$ ). Control animals only received vehicle solution mixed with their food $(2 \% \mathrm{v} / \mathrm{v} \mathrm{DMSO})$. Superoxide anion formation in response to $3 \mu \mathrm{g} / \mathrm{ml}$ collagen or $0.1 \mathrm{unit} / \mathrm{ml}$ thrombin) was measured for 10 minutes by EPR spectrometry (statistical analysis in Ai and representative examples in Aii and Aiii). Collagen- and thrombin-dependent aggregation were measured by turbidimetry (Bi and Bii, respectively). Statistical analysis was performed by one-way ANOVA with Bonferroni post-test $\left(\mathrm{n}=4,{ }^{*}\right.$ for $p<0.05$, ${ }^{* *}$ for $p<0.01,{ }^{* * *}$ for $\mathrm{p}<0.001$, ns for non-significant).

Figure 7: Administration of 2APT and 2APT-D6 reduces thrombus formation under flow and ferric chloride-induced carotid occlusionin vivo, but does not affect haemostasis in a tail tip transection assay. Drugs were administered in food (daily dose $=200 \mathrm{mg} / \mathrm{kg}$ ). Control animals only received vehicle solution mixed with their food $(2 \% \mathrm{v} / \mathrm{v}$ DMSO). Whole blood thrombus formation under shear stress (1,000 sec-1) was measured by DiOC6 platelet staining and fluorescence imaging (A). Representative pictures of thrombi after 10 minutes of flow are shown in $\mathrm{Ai}$, while quantification of the surface area coverage is shown in Aii. The time required for complete carotid occlusion was plotted for 
vehicle-, 2APT- and 2APT-D6-treated animals (Bi, $\mathrm{n}=6$, mean \pm SEM). Confirmation of carotid occlusion obtained by hematoxylin staining and imaging of carotid sections is shown in Bii. The same treatments were assessed in a tail tip transection assays in which the time required for full hamostasis (interruption of bleeding) was recorded $(\mathrm{C}, \mathrm{n}=9$, mean $\pm \mathrm{SEM})$. Statistical analysis was performed by one-way ANOVA with Bonferroni post-test ( $*$ for $p<0.05, * *$ for $p<0.01, * * *$ for $\mathrm{p}<0.001$, ns for non-significant).

Figure 8: Graphical interpretation of the data presented in this manuscript. 2APT (and 2APTD6 with higher potency) selectively inhibits NOX1 and collagen-dependent platelet activation, while sparing thrombin responses. In vivo, this translates in attenuation of thrombosis without effects on hemostasis.

\section{References}

Abubaker AA, Vara D, Eggleston I, Canobbio I, \& Pula G (2019). A novel flow cytometry assay using dihydroethidium as redox-sensitive probe reveals NADPH oxidase-dependent generation of superoxide anion in human platelets exposed to amyloid peptide beta. Platelets 30:181-189.

Akbar H, Duan X, Piatt R, Saleem S, Davis AK, Tandon NN, et al.(2018). Small molecule targeting the Rac1NOX2 interaction prevents collagen-related peptide and thrombin-induced reactive oxygen species generation and platelet activation. J Thromb Haemost 16:2083-2096.

Akbar H, Duan X, Saleem S, Davis AK, \& Zheng Y (2016). RhoA and Rac1 GTPases Differentially Regulate Agonist-Receptor Mediated Reactive Oxygen Species Generation in Platelets. PLoS One 11: e0163227.

Anvari E, Wikstrom P, Walum E, \& Welsh N (2015). The novel NADPH oxidase 4 inhibitor GLX351322 counteracts glucose intolerance in high-fat diet-treated C57BL/6 mice. Free Radic Res 49:1308-1318.

Arthur JF, Dunkley S, \& Andrews RK (2007). Platelet glycoprotein VI-related clinical defects. Br J Haematol 139: $363-372$.

Bach RD, \& Canepa C (1996). Electronic Factors Influencing the Decarboxylation of beta-Keto Acids. A Model Enzyme Study. J Org Chem 61: 6346-6353.

Bedard K, \& Krause KH (2007). The NOX family of ROS-generating NADPH oxidases: physiology and pathophysiology. Physiol Rev 87:245-313.

Begonja AJ, Gambaryan S, Geiger J, Aktas B, Pozgajova M, Nieswandt B, et al. (2005). Platelet NAD(P)Hoxidase-generated ROS production regulates alphaIIbbeta3-integrin activation independent of the NO/cGMP pathway. Blood 106: 2757-2760.

Bonnard T, \& Hagemeyer CE (2015). Ferric Chloride-induced Thrombosis Mouse Model on Carotid Artery and Mesentery Vessel. J Vis Exp:e52838.

Buresly K, Eisenberg MJ, Zhang X, \& Pilote L (2005). Bleeding complications associated with combinations of aspirin, thienopyridine derivatives, and warfarin in elderly patients following acute myocardial infarction. Arch Intern Med 165: 784-789.

Cammisotto V, Carnevale R, Nocella C, Stefanini L, Bartimoccia S, Coluccia A, et al. (2019). Nox2-mediated platelet activation by glycoprotein (GP) VI: Effect of rivaroxaban alone and in combination with aspirin. Biochem Pharmacol 163: 111-118.

Carnevale R, Loffredo L, Sanguigni V, Plebani A, Rossi P, Pignata C, et al. (2014). Different degrees of $\mathrm{NADPH}$ oxidase 2 regulation and in vivo platelet activation: lesson from chronic granulomatous disease. J Am Heart Assoc 3: e000920.

Chlopicki S, Olszanecki R, Janiszewski M, Laurindo FR, Panz T, \& Miedzobrodzki J (2004). Functional role of NADPH oxidase in activation of platelets. Antioxid Redox Signal 6: 691-698.

Csanyi G, Cifuentes-Pagano E, Al Ghouleh I, Ranayhossaini DJ, Egana L, Lopes LR, et al. (2011). Nox2 B-loop peptide, Nox2ds, specifically inhibits the NADPH oxidase Nox2. Free Radic Biol Med 51: 1116-1125. 
Dayal S, Wilson KM, Motto DG, Miller FJ, Jr., Chauhan AK, \& Lentz SR (2013). Hydrogen peroxide promotes aging-related platelet hyperactivation and thrombosis. Circulation 127: 1308-1316.

Delaney MK, Kim K, Estevez B, Xu Z, Stojanovic-Terpo A, Shen B, et al. (2016). Differential Roles of the NADPH-Oxidase 1 and 2 in Platelet Activation and Thrombosis. Arterioscler Thromb Vasc Biol 36:846-854.

El Haouari M (2019). Platelet Oxidative Stress and its Relationship with Cardiovascular Diseases in Type 2 Diabetes Mellitus Patients. Curr Med Chem 26: 4145-4165.

Florian P, Wonerow P, Harder S, Kuczka K, Dubar M, \& Graff J (2017). Anti-GPVI Fab SAR264565 effectively blocks GPVI function in ex vivo human platelets under arterial shear in a perfusion chamber. Eur J Clin Pharmacol 73: 949-956.

Gavazzi G, Banfi B, Deffert C, Fiette L, Schappi M, Herrmann F, et al. (2006). Decreased blood pressure in NOX1-deficient mice. FEBS Lett 580: 497-504.

Ghasemzadeh M, Hosseini E, Roudsari ZO, \& Zadkhak P (2018). Intraplatelet reactive oxygen species (ROS) correlate with the shedding of adhesive receptors, microvesiculation and platelet adhesion to collagen during storage: Does endogenous ROS generation downregulate platelet adhesive function? Thromb Res 163: 153161.

Gianni D, Taulet N, Zhang H, DerMardirossian C, Kister J, Martinez L, et al. (2010). A novel and specific NADPH oxidase-1 (Nox1) small-molecule inhibitor blocks the formation of functional invadopodia in human colon cancer cells. ACS Chem Biol 5: 981-993.

Gray SP, Di Marco E, Okabe J, Szyndralewiez C, Heitz F, Montezano AC, et al. (2013). NADPH oxidase 1 plays a key role in diabetes mellitus-accelerated atherosclerosis. Circulation 127:1888-1902.

Gray SP, Jha JC, Kennedy K, van Bommel E, Chew P, Szyndralewiez C, et al. (2017). Combined NOX1/4 inhibition with GKT137831 in mice provides dose-dependent reno- and atheroprotection even in established micro- and macrovascular disease. Diabetologia 60: 927-937.

Grover SP, \& Mackman N (2019). How useful are ferric chloride models of arterial thrombosis? Platelets: $1-7$.

Hirano K, Chen WS, Chueng AL, Dunne AA, Seredenina T, Filippova A, et al. (2015). Discovery of GSK2795039, a Novel Small Molecule NADPH Oxidase 2 Inhibitor. Antioxid Redox Signal 23: 358-374.

Jang JY, Min JH, Wang SB, Chae YH, Baek JY, Kim M, et al. (2015). Resveratrol inhibits collageninduced platelet stimulation through suppressing NADPH oxidase and oxidative inactivation of SH2 domaincontaining protein tyrosine phosphatase-2. Free Radic Biol Med 89: 842-851.

Jarvis GE, Raynal N, Langford JP, Onley DJ, Andrews A, Smethurst PA, et al. (2008). Identification of a major GpVI-binding locus in human type III collagen. Blood 111: 4986-4996.

Jiang JX, Chen X, Serizawa N, Szyndralewiez C, Page P, Schroder K, et al. (2012). Liver fibrosis and hepatocyte apoptosis are attenuated by GKT137831, a novel NOX4/NOX1 inhibitor in vivo. Free Radic Biol Med 53: 289-296.

Jiang P, \& Jandrot-Perrus M (2014). New advances in treating thrombotic diseases: GPVI as a platelet drug target. Drug Discov Today 19:1471-1475.

Krotz F, Sohn HY, Gloe T, Zahler S, Riexinger T, Schiele TM, et al. (2002). NAD(P)H oxidase-dependent platelet superoxide anion release increases platelet recruitment. Blood 100: 917-924.

Kwon G, Uddin MJ, Lee G, Jiang S, Cho A, Lee JH, et al. (2017). A novel pan-Nox inhibitor, APX-115, protects kidney injury in streptozotocin-induced diabetic mice: possible role of peroxisomal and mitochondrial biogenesis. Oncotarget 8: 74217-74232. 
Leppanen J, Savolainen J, Nevalainen T, Forsberg M, Huuskonen J, Taipale H, et al. (2001). Synthesis and in-vitro/in-vivo evaluation of orally administered entacapone prodrugs. J Pharm Pharmacol 53:1489-1498.

Magwenzi S, Woodward C, Wraith KS, Aburima A, Raslan Z, Jones H, et al. (2015). Oxidized LDL activates blood platelets through CD36/NOX2-mediated inhibition of the cGMP/protein kinase G signaling cascade. Blood 125: 2693-2703.

Massberg S, Konrad I, Bultmann A, Schulz C, Munch G, Peluso M, et al. (2004). Soluble glycoprotein VI dimer inhibits platelet adhesion and aggregation to the injured vessel wall in vivo. FASEB J 18:397-399.

Mojica Munoz AK, Jamasbi J, Uhland K, Degen H, Munch G, Ungerer M, et al. (2017). Recombinant GPVI-Fc added to single or dual antiplatelet therapy in vitro prevents plaque-induced platelet thrombus formation. Thromb Haemost 117: 1651-1659.

Monteiro PF, Morganti RP, Delbin MA, Calixto MC, Lopes-Pires ME, Marcondes S, et al. (2012). Platelet hyperaggregability in high-fat fed rats: a role for intraplatelet reactive-oxygen species production. Cardiovasc Diabetol 11: 5 .

Nagy M, van Geffen JP, Stegner D, Adams DJ, Braun A, de Witt SM, et al. (2019). Comparative Analysis of Microfluidics Thrombus Formation in Multiple Genetically Modified Mice: Link to Thrombosis and Hemostasis. Front Cardiovasc Med 6: 99.

Nieswandt B, Schulte V, Bergmeier W, Mokhtari-Nejad R, Rackebrandt K, Cazenave JP, et al. (2001). Long-term antithrombotic protection by in vivo depletion of platelet glycoprotein VI in mice. J Exp Med 193: 459-469.

Pollock JD, Williams DA, Gifford MA, Li LL, Du X, Fisherman J, et al. (1995). Mouse model of X-linked chronic granulomatous disease, an inherited defect in phagocyte superoxide production. Nat Genet 9: 202209.

Rezende F, Moll F, Walter M, Helfinger V, Hahner F, Janetzko P, et al. (2018). The NADPH organizers NoxO1 and p47phox are both mediators of diabetes-induced vascular dysfunction in mice. Redox Biol 15: $12-21$.

Rochat S, \& Alberio L (2015). Formaldehyde-fixation of platelets for flow cytometric measurement of phosphatidylserine exposure is feasible. Cytometry A 87: 32-36.

Sonkar VK, Kumar R, Jensen M, Wagner BA, Sharathkumar AA, Miller FJ, Jr., et al. (2019). Nox2 NADPH oxidase is dispensable for platelet activation or arterial thrombosis in mice. Blood Adv 3: 1272-1284.

Stef G, Csiszar A, Ziangmin Z, Ferdinandy P, Ungvari Z, \& Veress G (2007). Inhibition of NAD(P)H oxidase attenuates aggregation of platelets from high-risk cardiac patients with aspirin resistance. Pharmacol Rep 59: $428-436$.

Strassel C, Nonne C, Eckly A, David T, Leon C, Freund M, et al.(2007). Decreased thrombotic tendency in mouse models of the Bernard-Soulier syndrome. Arterioscler Thromb Vasc Biol 27:241-247.

Swan D, Loughran N, Makris M, \& Thachil J (2019). Management of bleeding and procedures in patients on antiplatelet therapy. Blood Rev: 100619.

Teixeira G, Szyndralewiez C, Molango S, Carnesecchi S, Heitz F, Wiesel P, et al. (2017). Therapeutic potential of NADPH oxidase 1/4 inhibitors. Br J Pharmacol 174: 1647-1669.

Vaezzadeh N, Ni R, Kim PY, Weitz JI, \& Gross PL (2014). Comparison of the effect of coagulation and platelet function impairments on various mouse bleeding models. Thromb Haemost 112: 412-418.

Vara D, Campanella M, \& Pula G (2013). The novel NOX inhibitor 2-acetylphenothiazine impairs collagendependent thrombus formation in a GPVI-dependent manner. Br J Pharmacol 168: 212-224. 
Vara D, \& Pula G (2014). Reactive oxygen species: physiological roles in the regulation of vascular cells. Curr Mol Med 14:1103-1125.

Violi F, \& Pignatelli P (2014). Platelet NOX, a novel target for anti-thrombotic treatment. Thromb Haemost 111: $817-823$.

Voors-Pette C, Lebozec K, Dogterom P, Jullien L, Billiald P, Ferlan P, et al. (2019). Safety and Tolerability, Pharmacokinetics, and Pharmacodynamics of ACT017, an Antiplatelet GPVI (Glycoprotein VI) Fab. Arterioscler Thromb Vasc Biol 39: 956-964.

Walsh TG, Berndt MC, Carrim N, Cowman J, Kenny D, \& Metharom P (2014). The role of Nox1 and Nox2 in GPVI-dependent platelet activation and thrombus formation. Redox Biol 2: 178-186.

Wang SB, Jang JY, Chae YH, Min JH, Baek JY, Kim M, et al. (2015). Kaempferol suppresses collageninduced platelet activation by inhibiting NADPH oxidase and protecting SHP-2 from oxidative inactivation. Free Radic Biol Med 83: 41-53.

Wingler K, Altenhoefer SA, Kleikers PW, Radermacher KA, Kleinschnitz C, \& Schmidt HH (2012). VAS2870 is a pan-NADPH oxidase inhibitor. Cell Mol Life Sci 69: 3159-3160.

Yasuda H, Matsuo Y, Sato Y, Ozawa S, Ishigooka S, Yamashita M, et al. (2015). Treatment and prevention of gastrointestinal bleeding in patients receiving antiplatelet therapy. World J Crit Care Med 4: 40-46.

Youn JY, Gao L, \& Cai H (2012). The p47phox- and NADPH oxidase organiser 1 (NOXO1)-dependent activation of NADPH oxidase 1 (NOX1) mediates endothelial nitric oxide synthase (eNOS) uncoupling and endothelial dysfunction in a streptozotocin-induced murine model of diabetes. Diabetologia 55: 2069-2079. 


\begin{tabular}{|c|c|}
\hline & $\begin{array}{c}\text { Molecular } \\
\text { Weight }(\mathrm{g} / \mathrm{mol})\end{array}$ \\
\hline 2APT & 241.31 \\
\hline 2APT-D1 & 299.34 \\
\hline 2APT-D2 & 313.37 \\
\hline 2APT-D3 & 243.32 \\
\hline 2APT-D4 & 313.37 \\
\hline 2APT-D5 & 284.33 \\
\hline 2APT-D6 & 341.43 \\
\hline 2APT-D7 & 319.38 \\
\hline 2APT-D8 & 329.37 \\
\hline 2APT-D9 & 375.44 \\
\hline
\end{tabular}

Table 1: 2APT derivative structures. 
A)

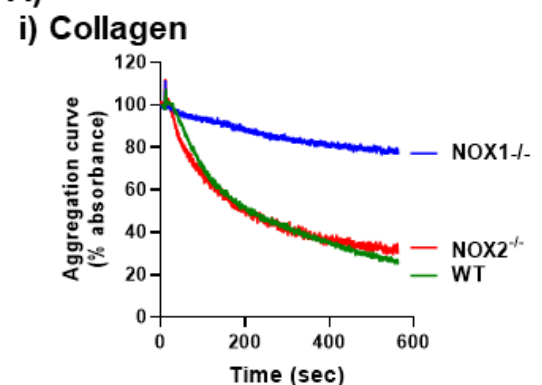

B)

i)

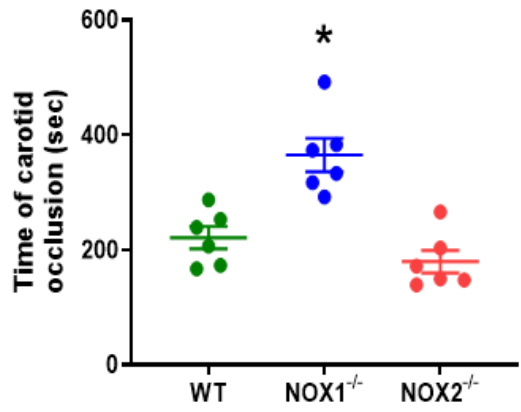

C)

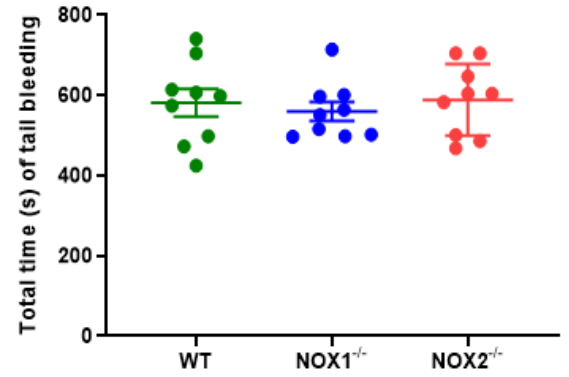

ii) Thrombin

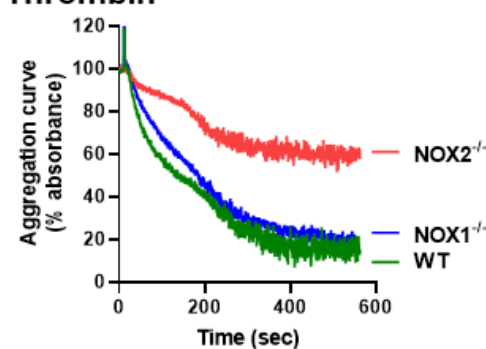

ii)

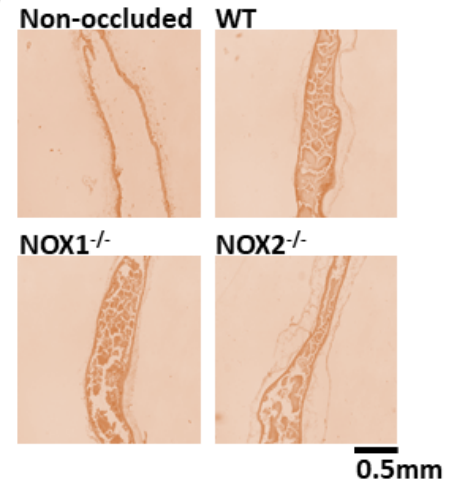

Figure 1 
A) EPR, Collagen
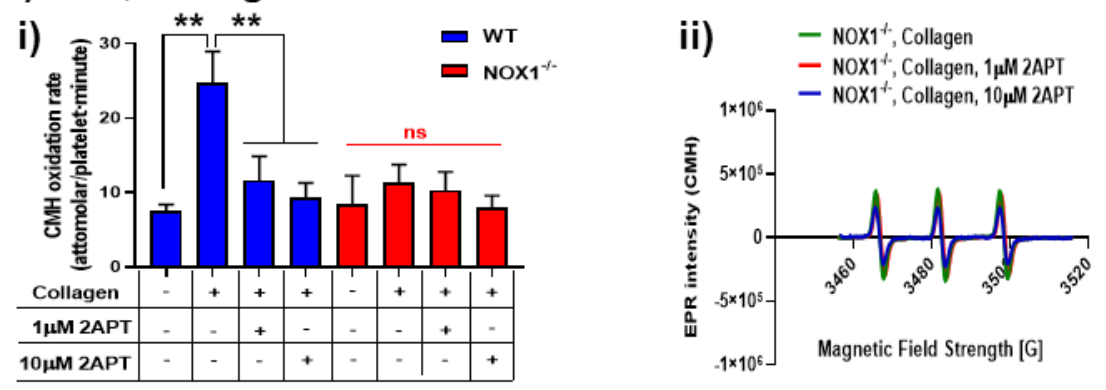

\section{B) EPR, Thrombin}

i)

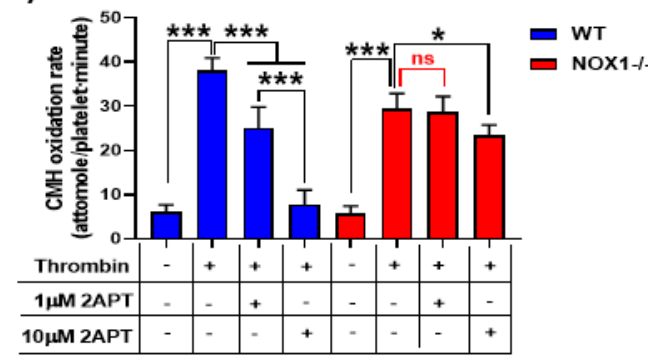

ii) $\quad-$ Noxi $^{-1}$, Thrombin

- NOX $1^{-1}$, Thrombin, $1 \mu \mathrm{M}$ 2APT

- NOX1 ${ }^{-t}$, Thrombin, $10 \mu \mathrm{M}$ 2APT

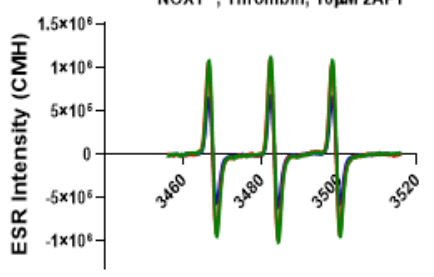

Magnetic Field strenght (G)

\section{C) Aggregation, Collagen}

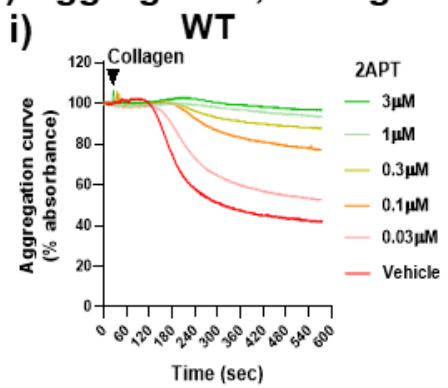

\section{D) Aggregation, Thrombin}

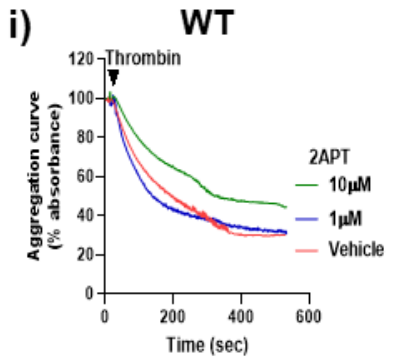

Figure 2
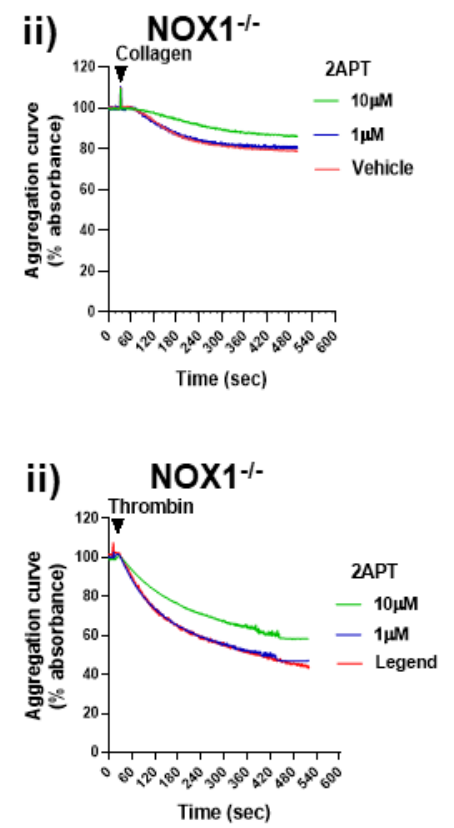
A) Adhesion, Collagen

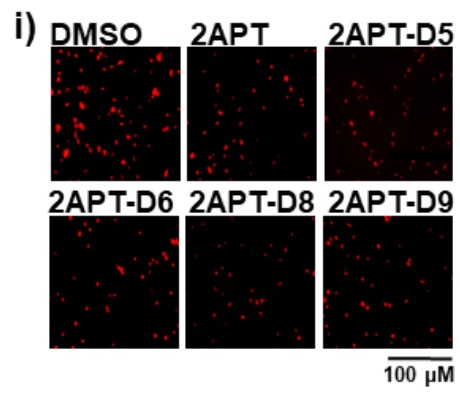

B) Adhesion, Fibrinogen

i) DMSO 2APT 2APT-D5

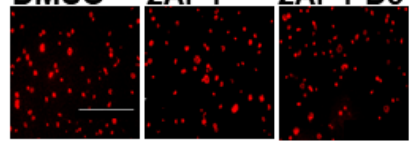

2APT-D6 2APT-P8 2APT-D9

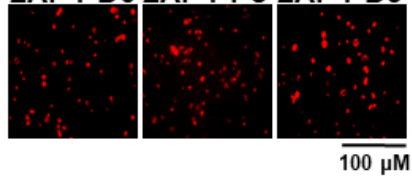

C) Aggregation, Collagen

i)

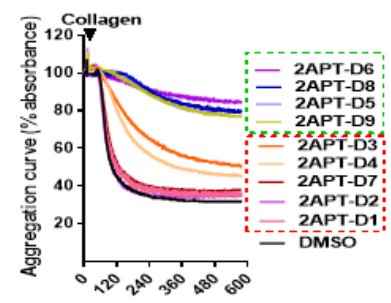

D) Aggregation, Thrombin

i)

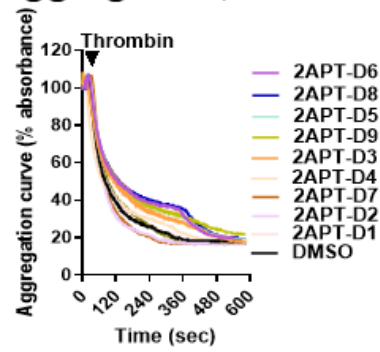

Figure 3 ii)

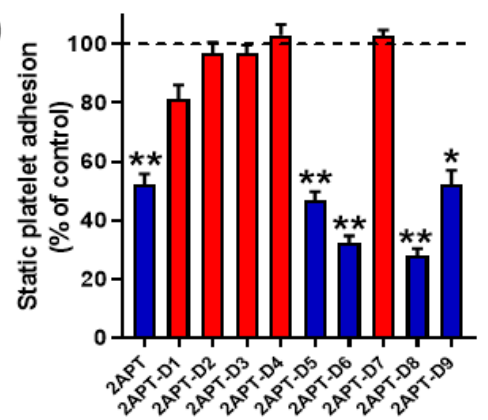

ii)

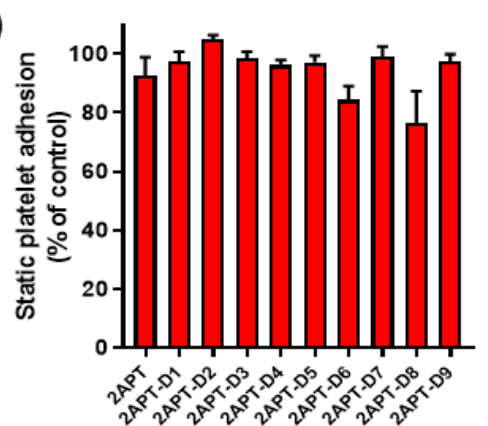

ii)

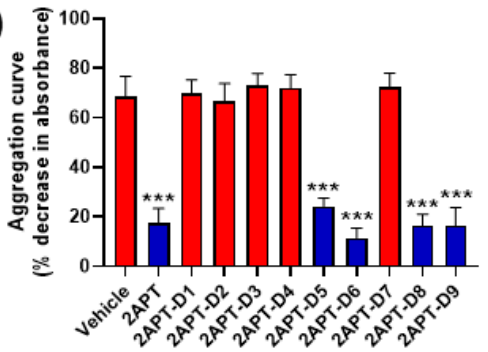

ii)

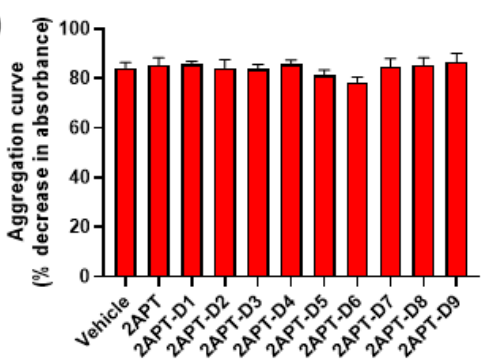




\section{A) EPR, Collagen}
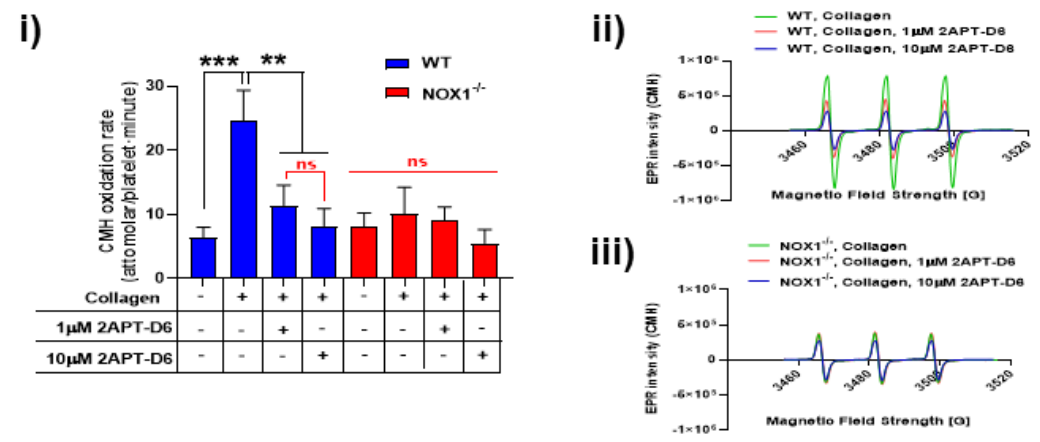

\section{B) EPR, Thrombin}
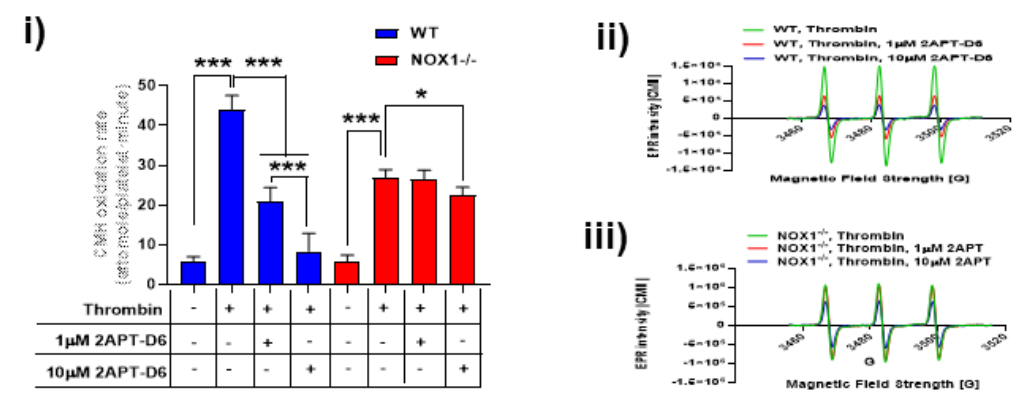

\section{C) Aggregation, Collagen}
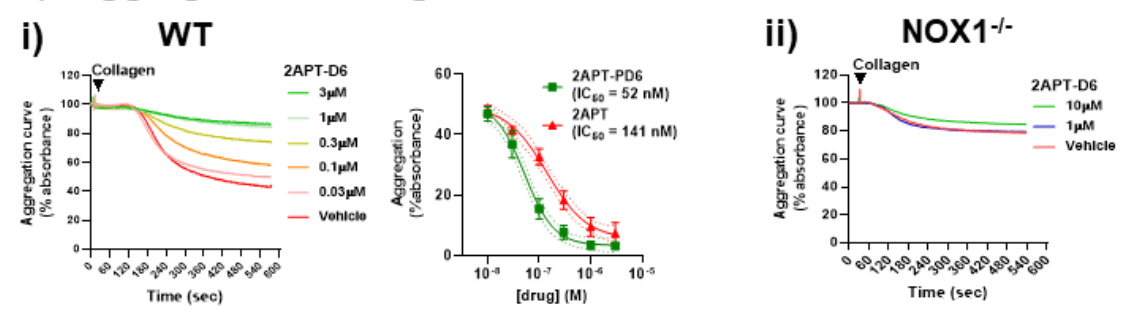

\section{D) Aggregation, CRP-XL}

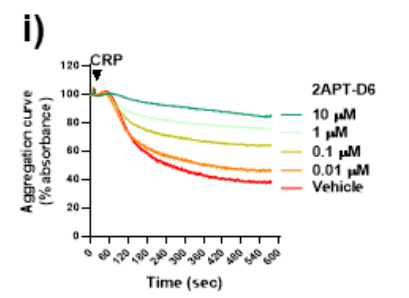

Figure 4
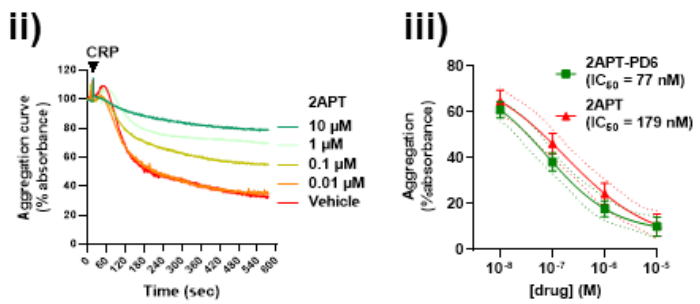
A) Thrombus formation, Collagen

i)
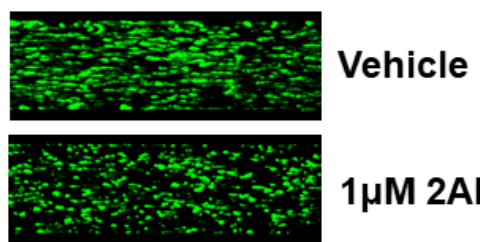

$1 \mu \mathrm{M} 2 \mathrm{APT}$

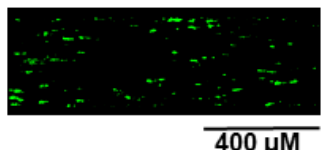

$1 \mu \mathrm{M}$ 2APT-D6

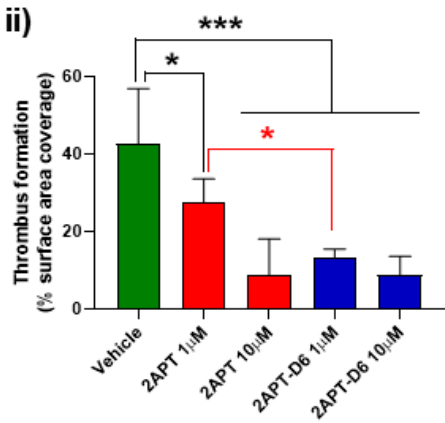

B) Flow cytometry
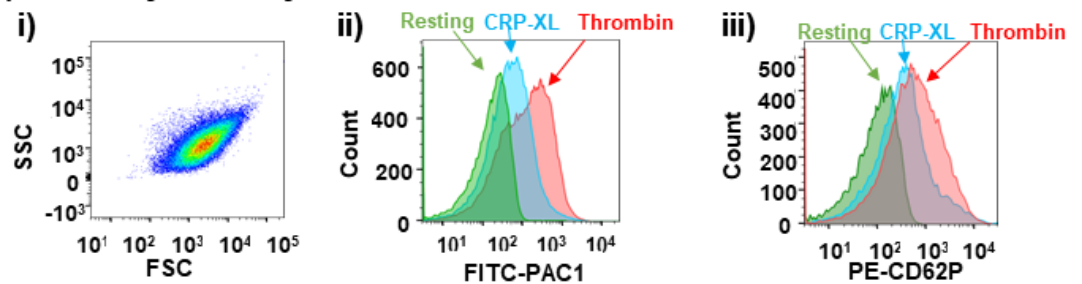

C) Flow cytometry, integrin allb $\beta 3$ activation

i) CRP-XL

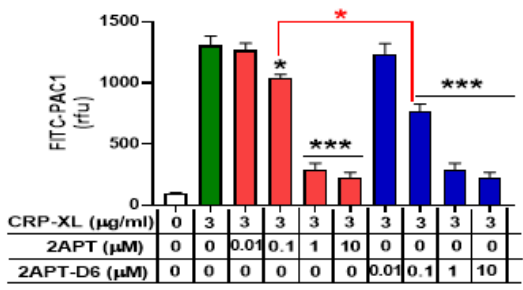

ii) Thrombin

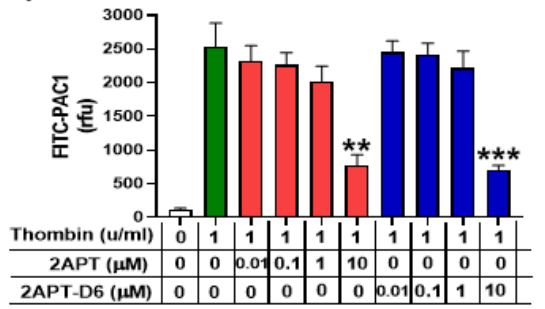

D) Flow cytometry, P-selectin externalization

i) CRP-XL

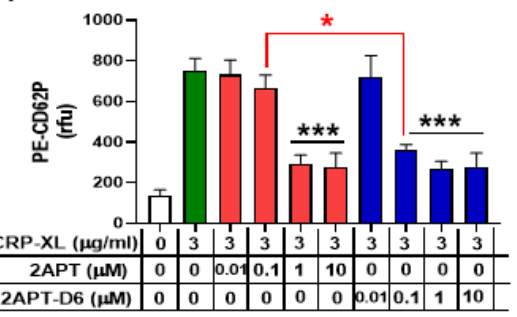

ii) Thrombin

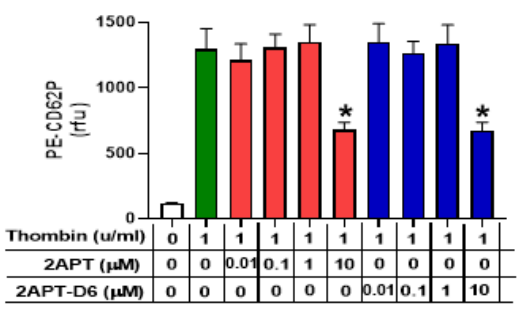

Figure 5 
A) EPR

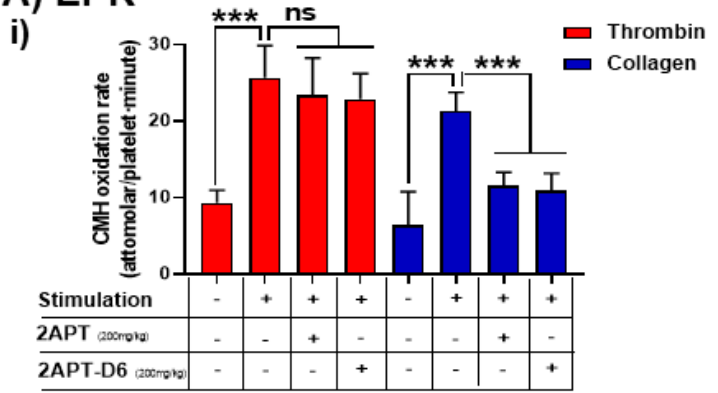

ii) Collagen

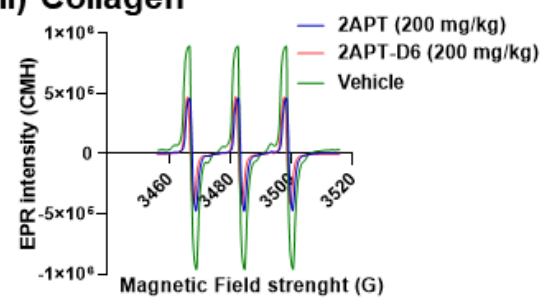

\section{B) Aggregation}

\section{i) Collagen}

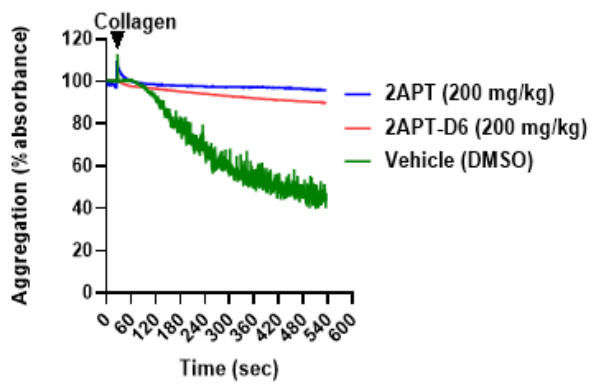

iii) Thrombin

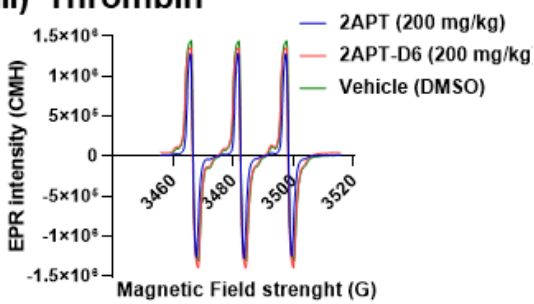

Figure 6

ii) Thrombin

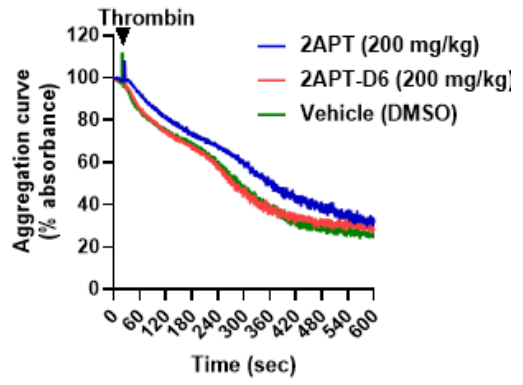


A) Thrombus formation, Collagen

i)

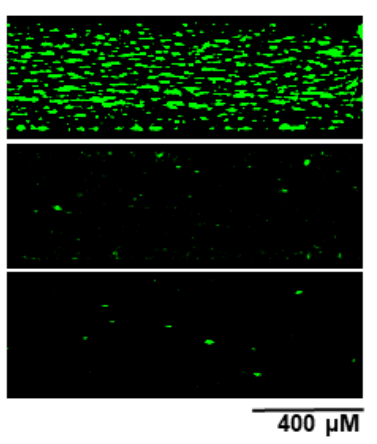

B)

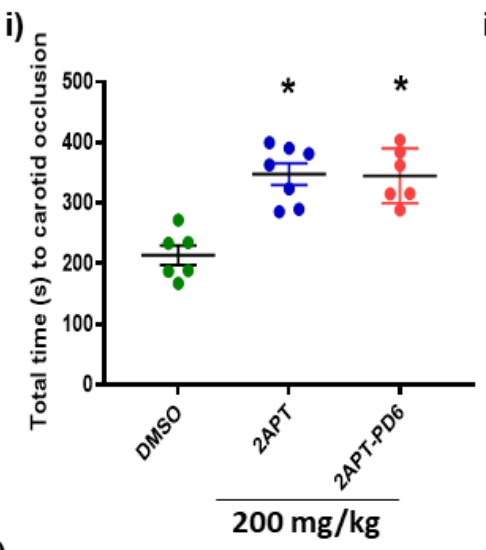

C)

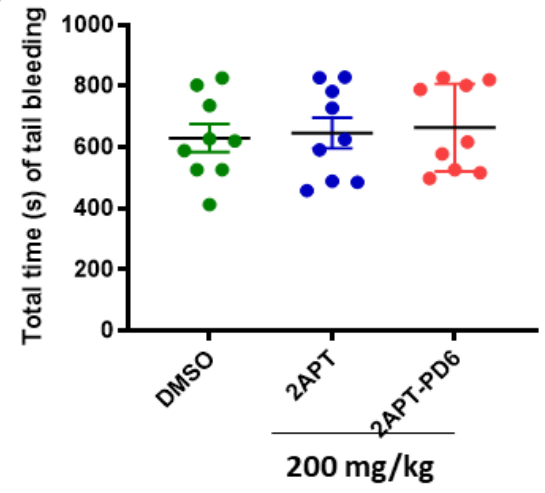

ii)

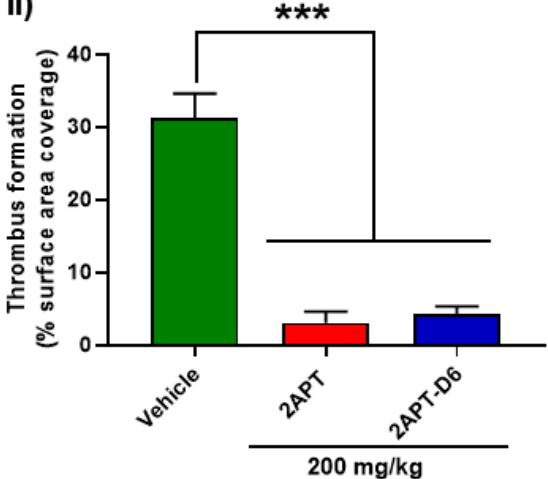

ii) Non-occluded DMSO

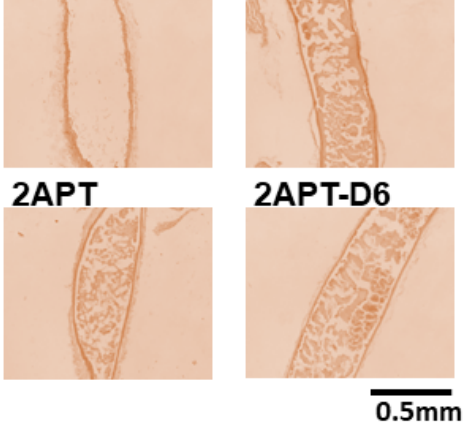

Figure 7 


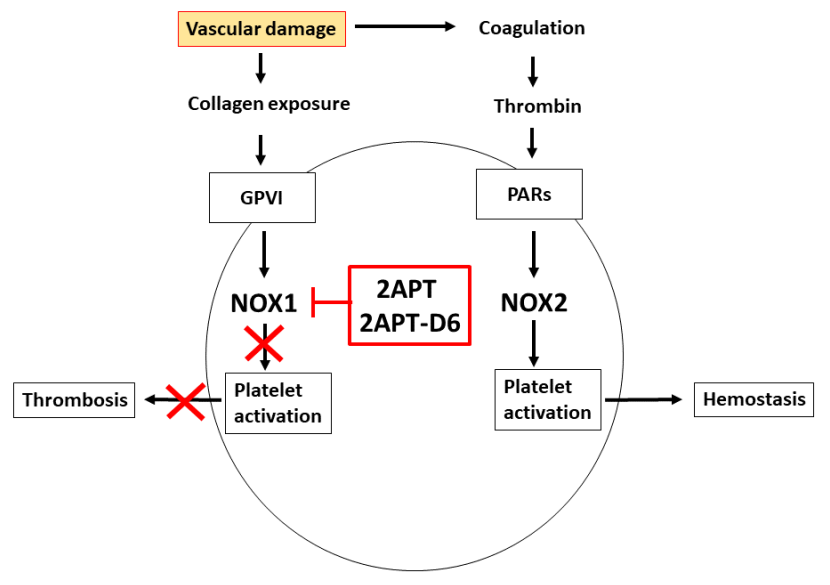

